\title{
Author Correction: EGFR and HER2 exon 20 insertions in solid tumours: from biology to treatment
}

Alex Friedlaender, Vivek Subbiah (D), Alessandro Russo, Giuseppe Luigi Banna, Umberto Malapelle, Christian Rolfo and Alfredo Addeo (i)

Correction to: Nature Reviews Clinical Oncology https://doi.org/10.1038/s41571-021-00558-1, published online 24 September 2021.

In the original version of this article, the affiliation of Giuseppe Luigi Banna was incomplete and should have read 'Candiolo Cancer Institute, FPO-IRCCS, Candiolo, Italy'. In addition, 'Chomosome' was misspelled in figures 1 and 3. These errors have now been corrected in the HTML and PDF versions of the article.

https://doi.org/10.1038/s41571-021-00571-4 I Published online 5 October 2021

๑) Springer Nature Limited 2021 